\title{
BAJOS IMPUESTOS AL SUELO E INOPERANCIA DEL INSTRUMENTO TRIBUTARIO*
}

Edna Cristina Bonilla Sebáa

"DOI: https://doi.org/10.18601/01245996.v20n39.09. Este artículo resume apartes de dos capítulos de la tesis La tributación al suelo: expresión de la regresividad y de la inequidad en Colombia (1914-2014) para obtener el doctorado en Estudios Políticos, Universidad Externado de Colombia. Recepción: 03-04-2018, modificación final: 08-05-2018, aceptación: 11-052018. Sugerencia de citación: Bonilla S., E. C. (2018). Bajos impuestos al suelo e inoperancia del instrumento tributario. Revista de Economía Institucional, 20(39), 211-229.

a Doctora en Estudios Políticos. Profesora asociada, Facultad de Ciencias Económicas, Universidad Nacional de Colombia, Bogotá, Colombia, [ecbonillas@unal.edu.co] 


\section{Bajos impuestos al suelo e inoperancia del instrumento tributario}

Resumen. Este artículo muestra que los tributos al suelo, en especial el impuesto predial, son muy bajos en Colombia, a pesar de la potencialidad fiscal de los municipios. Los avalúos están atrasados y, aun así, la tarifa efectiva del impuesto predial no llega al 3 por mil. Además, la estructura de estos impuestos es muy desordenada, y varía considerablemente entre municipios. Muestra la poca relevancia de los impuestos como instrumento para modificar las relaciones factoriales y mejorar la eficiencia de la producción, sobre todo en el campo. Con base en los datos del Censo Nacional Agropecuario 2014 constata la alta concentración de la tenencia de tierra, en área y en unidades productivas, y así como la importancia de la pequeña producción agropecuaria. Señala, además, que el diseño de los impuestos al suelo no contribuye a mejorar la productividad agropecuaria.

Palabras clave: política fiscal, impuesto predial, productividad agropecuaria, distribución del ingreso y de la riqueza; JEL: E61, E62, H25, H26.

\section{Low land taxes and ineffectiveness of the tax system}

Abstract. This article shows that taxes on land, especially the property tax, are very low in Colombia, despite the fiscal potential of the municipalities. The appraisals are out of date and even so, the effective rate of property tax does not reach three per thousand. In addition, the structure of these taxes is very disorganized, and varies considerably among municipalities. It shows the slight relevance of taxes as an instrument to modify factorial relationships and improve the efficiency of production, especially in the countryside. Based on the data of the National Agricultural Census 2014 , it finds a high concentration of land tenure, in terms of area and productive units, and the importance of small agricultural production. It also points out that the design of land taxes does not contribute to improving agricultural productivity.

Keywords: Fiscal policy, property tax, agricultural productivity, distribution of income and wealth; JEL: E61, E62, H25, H26.

\section{Baixos impostos sobre a terra e inoperância do instrumento tributário}

Resumo. Este artigo mostra que as tributações do solo, em especial o imposto predial, são muito baixas na Colômbia, apesar da potencialidade fiscal dos municípios. As avaliações estão atrasadas e, ainda assim, a taxa efetiva do imposto predial não chega a $0,3 \%$. Além disso, a estrutura desses impostos é muito desorganizada e varia de forma considerável entre os municípios. Mostra-se a pouca relevância da produção, principalmente no campo. Com base nos dados do censo Nacional Agropecuário 2014, constata-se a alta concentração da posse de terra, em área e unidades produtivas, bem como a importância da pequena produção agropecuária. Além disso, o texto indica que o desenho dos impostos do solo não contribui para melhorar a produtividade agropecuária.

Palavras-chaves: política fiscal, imposto predial, produtividade agropecuária, distribuição da renda e da riqueza; JEL: E61, E62, H25, H26. 
- 1 artículo comienza con una presentación de la concepción de CHenry George sobre los impuestos a la tierra. En la segunda sección se muestran los datos del censo agropecuario sobre la concentración de la tierra y el predominio de la pequeña producción. En la tercera sección se pone en evidencia que la tarifa efectiva del impuesto predial es baja. En la sección final se argumenta que en Colombia el instrumento tributario no se ha utilizado para conciliar la vocación y el uso del suelo, para crear incentivos que favorezcan a la pequeña producción agropecuaria ni para mejorar la equidad tributaria.

\section{HENRY GEORGE Y LOS IMPUESTOS AL SUELO}

Exceptuando las cuatro grandes ciudades del país, los tributos al suelo y en particular el impuesto predial tienen dos características generales: son bajos e inequitativos. Esta afirmación adquiere especial validez en las áreas rurales.

La forma como ha evolucionado la tributación al suelo entre 1914 y 2014 se caracteriza por su regresividad, la cual ha contribuido a consolidar una sociedad profundamente inequitativa. La tributación es un tema político crucial. Toca a toda la sociedad, y cuando se plantea buscar la equidad necesariamente se debe pasar por el régimen impositivo. Desde los escritos de los autores clásicos hasta los trabajos más recientes en economía y política se ha reconocido la importancia de la tributación. En el siglo XIX, los autores clásicos hacían especial énfasis en los impuestos al suelo.

La discusión sobre los impuestos como instrumento de intervención del Estado obliga a encontrar un acuerdo político sobre la forma de tributación que se considera más pertinente. Es inevitable que todas las soluciones que se propongan sean subóptimas desde diversos puntos de vista.

Quizá el punto de referencia más importante en la discusión sobre el papel de los impuestos al suelo sea George (1982). Desde el inicio de su carrera política, Henry George se interesó por la pobreza y los diversos medios para superarla. Sus viajes por el mundo y su experiencia como hijo de una familia de clase media baja lo llevaron a concluir que gran parte del problema radicaba en las restricciones de acceso de la población a los medios básicos de supervivencia: trabajo y tierra. George observó que ambos elementos están íntimamente relacionados. Reconociendo que cada individuo es autónomo en el uso de su fuerza productiva, consideraba que la única manera para que el trabajo generara algún resultado era cuando se aplica a la trans- 
formación de la tierra. La acción estatal debía llevarse a cabo de un modo que no restringiera el acceso a la tierra porque limitaría el uso de la mano de obra. Pero tampoco se podía caer en el otro extremo, puesto que el acceso irrestricto a la tierra tenía muchas dificultades prácticas. La tributación ayudaba a encontrar un equilibrio razonable entre ambos extremos.

George siempre se consideró un liberal radical, y no estuvo de acuerdo con las propuestas comunistas que impulsaban la propiedad colectiva de la tierra. Como buen liberal, defendía la propiedad privada, pero exigía que la sociedad pudiera participar de las rentas. Siempre luchó contra los terratenientes porque le parecía injusto que se apropiaran de la renta diferencial sin trabajar. Para poder acabar con los terratenientes y lograr una sociedad más igualitaria, propuso que el impuesto a las rentas diferenciales fuera del 100\%.

$\mathrm{Su}$ argumento se puede dividir en tres puntos esenciales. Primero, puesto que la tierra es el factor de producción primario por excelencia, los impuestos a la tierra tienen impacto sobre las relaciones factoriales de toda la economía. Segundo, los impuestos al suelo favorecen la justicia. Desde este punto de vista, le parecía que los sistemas de apropiación de la tierra en Irlanda, Estados Unidos y otros "países civilizados", eran "atroces". Lo peor es que tal desorden se consideraba "natural” y "justo" (George, 1982, p. 8). Y, tercero, las contribuciones sobre la tierra son consistentes con los principios de una sociedad liberal y favorecen la equidad.

Aunque George percibió que la importancia de la tierra como factor productivo estaba en declive, criticó el desconocimiento del papel fundamental de la tierra en numerosos análisis económicos. En sus palabras: "La tierra es necesaria para toda la producción, sin importar su tipo o forma. La tierra es el único medio a través del que los seres humanos pueden acceder al universo material” (ibíd., p. 23). Desde una perspectiva contemporánea, dice Stiglitz "si los rendimientos de la tierra fueran gravados totalmente (como se haría con el impuesto de Henry George), la riqueza y el capital estarían totalmente alineados" (Stiglitz, 2015, p. 4).

La postura de George era consistente con los análisis de los autores clásicos. Smith (1994), Ricardo (2001), Marx (1968) y Mill (1978), observaron que el proceso de producción requiere tres factores (tierra o recursos naturales, capital y trabajo) y reconocieron las características especiales de la tierra: su oferta es fija y no implica costos de producción. Dadas estas características, si todo lo demás permanece constante, un aumento de la demanda de tierra se refleja en un mayor 
precio. El propietario de un inmueble cuya demanda aumenta es un privilegiado, puesto que en virtud de su derecho de propiedad puede disfrutar de un excedente que no es el resultado de su trabajo. Los clásicos definieron ese ingreso adicional como renta: el ingreso que se obtiene por la posesión de un bien escaso o un factor de producción exclusivo.

Los impuestos al suelo tienen varias ventajas. Son una fuente de recursos para los gobiernos locales, incentivan el uso eficiente del suelo y desestimulan la concentración improductiva de la tierra (Corbacho, Fretes y Lora, 2013; Banco Mundial, 2004). Además, actúan como reguladores de las relaciones factoriales. Hotelling (1931) mostró la capacidad de los tributos para modificar las relaciones tecnológicas, y en los temas ambientales para regular el ritmo de explotación de los recursos. Observó que hay una relación directa entre la velocidad de explotación de los recursos naturales y su tasa de rendimiento. Un aumento de los impuestos disminuye la tasa de rendimiento de la explotación minera y desestimula la explotación improductiva. La constatación de Hotelling es relevante porque en Colombia los impuestos al suelo, sobre todo en las zonas rurales, se han utilizado muy poco para mover las relaciones factoriales. En las ciudades grandes y en algunas intermedias, los prediales altos a los lotes de engorde obligan a que los propietarios vendan o construyan.

En suma, los impuestos al suelo deben cumplir el triple propósito de mejorar el recaudo, la equidad y la eficiencia (relaciones factoriales). Y la calibración de estos propósitos depende de que el suelo esté más relacionado con procesos urbanísticos o con dinámicas productivas.

\section{LA CONCENTRACIÓN DE LATIERRA}

En Colombia las políticas fiscales -la conjunción de los impuestos y de los subsidios- no han logrado modificar el índice de Gini. La concentración del ingreso es muy alta. Y la inequidad en la distribución de la tierra es más aguda que en el ingreso.

E1 cuadro 1 muestra la distribución de las unidades productoras agropecuarias (UPA), de las áreas y de los productores.

Las características de la distribución de los productores y de las áreas rurales se observan en la gráfica 1. En la parte superior se diferencian dos distribuciones según el tamaño de la UPA. Una corresponde a la de los productores (barras, eje izquierdo) y otra a la del área (puntos, eje derecho). E1 70,8\% de los productores están vinculados a UPA menores de 5 ha, que ocupan el 3,1\% del área censada. En 
las UPA de más de 1.000 ha se ubica el $0,2 \%$ de los productores, y ocupan el 59,5\% del área ${ }^{1}$. En el extremo superior hay 483 UPA mayores de 10.000 ha. Machado (2009) observa que la forma de las dos distribuciones es bimodal, ya que muy pocos productores ocupan la mayoría del área, y muchos productores ocupan un área reducida ${ }^{2}$. Esa observación ha sido confirmada por otros estudios realizados en el país, como el del PNUD (2011).

Cuadro 1

Número de productores y área ocupada por tamaño de la UPA

\begin{tabular}{lrrrrrrrrr}
\hline Tamaño en ha & \multicolumn{3}{c}{ UPA } & \multicolumn{4}{c}{ \% } & \multicolumn{3}{c}{ Área } & \multicolumn{3}{c}{ Productores } \\
\hline & Número & \multicolumn{1}{c}{$\%$} & \multicolumn{1}{c}{ ac. } & \multicolumn{1}{c}{$\%$} & $\%$ ac. & Número & $\%$ & $\%$ ac. \\
\hline$<0,5$ & 531.119 & 26,1 & 26,1 & 108.037 & 0,2 & 0,2 & 777.493 & 24,3 & 24,3 \\
$>0,5-1$ & 259.544 & 12,8 & 38,9 & 190.197 & 0,3 & 0,5 & 331.123 & 10,3 & 34,6 \\
$>1-2,5$ & 381.884 & 18,8 & 57,7 & 625.561 & 1,0 & 1,5 & 537.721 & 16,8 & 51,4 \\
$>2,5-3$ & 74.273 & 3,7 & 61,3 & 203.972 & 0,3 & 1,9 & 112.653 & 3,5 & 54,9 \\
$>3-5$ & 191.970 & 9,4 & 70,8 & 748.255 & 1,2 & 3,1 & 311.916 & 9,7 & 64,6 \\
$>5-10$ & 212.884 & 10,5 & 81,2 & 1.512 .323 & 2,5 & 5,6 & 368.442 & 11,5 & 76,1 \\
$>10-20$ & 152.154 & 7,5 & 88,7 & 2.149 .286 & 3,5 & 9,1 & 280.659 & 8,8 & 84,9 \\
$>20-50$ & 125.733 & 6,2 & 94,9 & 3.935 .925 & 6,5 & 15,6 & 246.571 & 7,7 & 92,6 \\
$>50-100$ & 52.894 & 2,6 & 97,5 & 3.696 .241 & 6,1 & 21,7 & 114.555 & 3,6 & 96,1 \\
$>100-200$ & 26.727 & 1,3 & 98,8 & 3.692 .677 & 6,1 & 27,7 & 62.857 & 2,0 & 98,1 \\
$>200-500$ & 15.169 & 0,7 & 99,6 & 4.566 .967 & 7,5 & 35,3 & 38.499 & 1,2 & 99,3 \\
$>500-1.000$ & 4.601 & 0,2 & 99,8 & 3.196 .841 & 5,3 & 40,5 & 12.662 & 0,4 & 99,7 \\
$>1.000-2.000$ & 2.128 & 0,1 & 99,9 & 2.928 .544 & 4,8 & 45,3 & 5.487 & 0,2 & 99,9 \\
$>2.000-5.000$ & 1.175 & 0,1 & 100,0 & 3.612 .532 & 5,9 & 51,3 & 2.685 & 0,1 & 99,9 \\
$>5.000-10.000$ & 424 & 0,0 & 100,0 & 2.833 .886 & 4,7 & 55,9 & 1.439 & 0,0 & 100,0 \\
$>10.000$ & 483 & 0,0 & 100,0 & 26.769 .566 & 44,1 & 100,0 & 270 & 0,0 & 100,0 \\
Total & 2.033 .162 & 100,0 & & 60.770 .811 & 100,0 & & 3.205 .032 & 100,0 & \\
\hline
\end{tabular}

No se incluyen comunidades étnicas ni parques.

Fuente: Dane, Censo Nacional Agropecuario de 2014, estimaciones de la Upra.

El coeficiente de Gini de propiedad de la tierra es, en promedio, de 0,81, pero en algunos departamentos, como en Antioquia, es de 0,91 (ibíd.). Durante los dos periodos de gobierno del presidente Uribe, el Gini aumentó. Y durante el gobierno del presidente Santos la concentración no disminuyó. De acuerdo con estimaciones de la Upra, los resultados del censo agropecuario muestran que la distribución ha empeorado. El coeficiente de Gini por área de las UPA es 0,931.

De acuerdo con el censo agropecuario de 2104, el 70,1\% de las UPA es de propiedad privada (y cubre el 36,6\% del área), el 12,5\% está en arriendo (y comprende el 3,6\% del área), y el 17,1\% no declara la forma de propiedad. La indeterminación de la propiedad es mayor

${ }^{1}$ En estos análisis de distribución se debe tener presente que no hay relación entre la UPA y el predio; así, nada se puede afirmar sobre la distribución de la propiedad.

2 Arteaga, Castro et al. (2016) adoptan otro enfoque y afirman que la distribución de la propiedad es multimodal. Muestran que en la historia del país la distribución de baldíos ha sido generosa y ha contribuido a mitigar la concentración de la tierra. 
en las UPA superiores a 1.000 ha. Y entre las de más de 10.000 ha, el 86,5\% (422 UPA de 483), no se reporta el tipo de propiedad. El arriendo se presenta especialmente en Boyacá, Cundinamarca, Antioquia y Nariño.

Gráfica 1

Participación de los productores y área ocupada por tamaño de la UPA Tendencias, total nacional

a. Distribución de los productores y del área

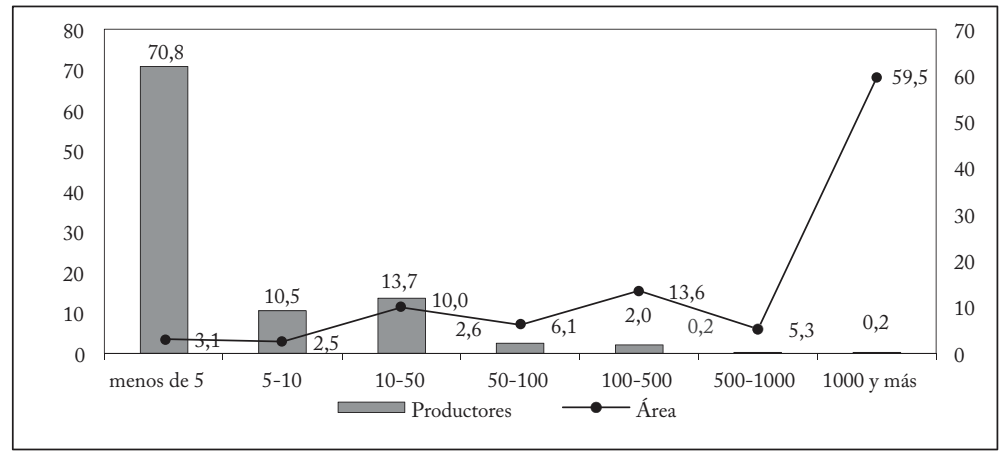

b. Tendencias de la distribución de los productores y del área

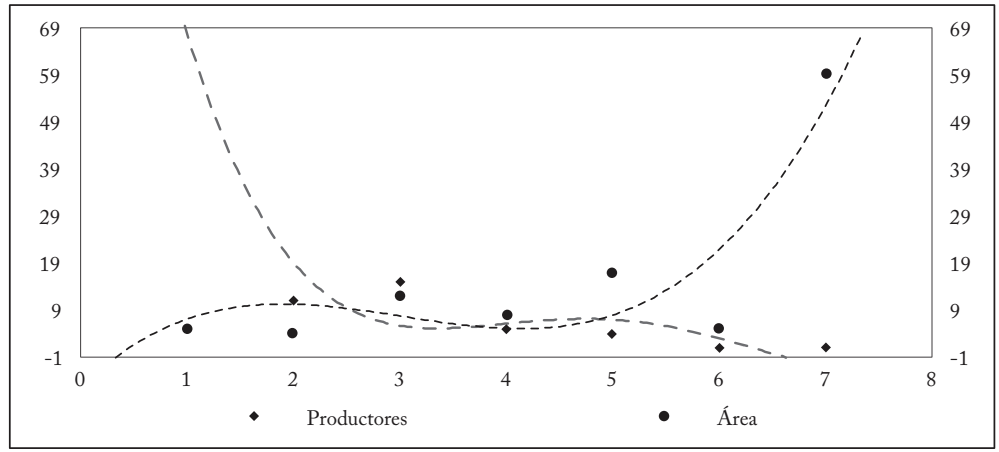

El eje horizontal corresponde al tamaño de la UPA en hectáreas. El eje vertical izquierdo mide la participación del área censada, y el eje vertical derecho la participación de los productores.

Fuente: CNA 2014, estimaciones de la Upra; datos del cuadro 1.

La alta concentración ha ido a la par de una predominancia de la pequeña unidad productiva. La tributación debe cumplir un doble papel. Por un lado, cambiar la distribución y, por el otro, incentivar el desarrollo de las pequeñas y medianas unidades de producción.

Para modificar una concentración de la tierra tan alta como la que se observa en el país, habría dos opciones de política pública. Por un lado, afectar directamente la distribución a través de mecanismos como 
la expropiación o la extinción de dominio. La otra vía, indirecta, es recurrir a incentivos tributarios. Si aumentan los impuestos a las tierras ociosas se obliga a que el propietario mejore la productividad. Si no lo hace, tiene que vender el predio porque el monto del impuesto se convierte en una carga insostenible. Esta segunda vía favorece la modernización de la producción agropecuaria y la dinamización del mercado de tierras.

\section{LAS TARIFAS EFECTIVAS DE LOS IMPUESTOS PREDIALES}

La descentralización le ha asignado responsabilidades fiscales a las entidades territoriales, sobre todo a los municipios. Sin embargo, la autonomía territorial se ha limitado porque los municipios no han aprovechado las potencialidades fiscales de los impuestos al suelo y al urbanismo. En general, los municipios han subestimado los instrumentos fiscales que les ofrece la Ley 388 de 1997. Hoy, una proporción significativa de los ingresos municipales proviene de las transferencias del gobierno nacional. La dependencia fiscal, especialmente en el caso de los departamentos, se ha agudizado con el aumento de las transferencias provenientes de las regalías.

Cuadro 2

Composición de los ingresos municipales, total nacional (Miles de millones de pesos corrientes)

\begin{tabular}{lrrrrrr}
\hline & \multicolumn{2}{c}{2009} & \multicolumn{2}{c}{2012} & \multicolumn{2}{c}{2015} \\
\hline & \multicolumn{1}{c}{ Valor } & \multicolumn{1}{c}{$\%$} & \multicolumn{1}{c}{ Valor } & \multicolumn{1}{c}{$\%$} & \multicolumn{1}{c}{ Valor } & \multicolumn{1}{c}{$\%$} \\
\hline Ingresos tributarios & $10.396,5$ & 30,7 & $13.504,3$ & 31,8 & $19.644,0$ & 31,5 \\
Predial & $3.076,4$ & 9,1 & $4.101,5$ & 9,7 & $6.364,0$ & 10,2 \\
Industria y comercio & $4.275,2$ & 12,6 & $5.666,1$ & 13,4 & $7.328,0$ & 11,8 \\
Sobretasa a la gasolina & $1.076,3$ & 3,2 & $1.141,4$ & 2,7 & $1.376,0$ & 2,2 \\
Otros & $1.968,7$ & 5,8 & $2.595,3$ & 6,1 & $4.576,0$ & 7,3 \\
Ingresos no tributarios & $1.752,2$ & 5,2 & $3.011,2$ & 7,1 & $3.063,0$ & 4,9 \\
Transferencias funcionamiento & $1.011,0$ & 3,0 & $1.281,5$ & 3,0 & $1.632,0$ & 2,6 \\
Ingresos de capital & $20.695,2$ & 61,1 & $24.627,0$ & 58,1 & $37.983,0$ & 60,9 \\
Regalías & $1.810,7$ & 5,4 & $1.333,4$ & 3,1 & $3.165,0$ & 5,1 \\
Transferencias (SGP y otros) & $15.317,5$ & 45,3 & $19.948,4$ & 47,0 & $27.072,0$ & 43,4 \\
Cofinanciación & 703,2 & 2,1 & 890,2 & 2,1 & $1.510,0$ & 2,4 \\
Otros & $2.863,8$ & 8,5 & $2.455,1$ & 5,8 & $6.236,0$ & 10,0 \\
Ingresos totales & $33.855,0$ & 100,0 & $42.423,8$ & 100,0 & $62.332,00$ & 100,0 \\
\hline
\end{tabular}

Fuente: DNP (2016).

La tributación es muy baja y desigual entre municipios. En 2015, del total de ingresos municipales de todo el país, el 31,5\% fue de origen tributario. Y alrededor del 30\% de los ingresos tributarios correspondía a recaudos del impuesto predial unificado. El impuesto predial solo aportó el 10,2\% de los ingresos municipales totales. Es claro, enton- 
ces, que la tributación al suelo no ha sido una fuente importante de ingresos para los municipios.

Pese a las limitaciones señaladas, la participación del impuesto predial en los ingresos totales viene en aumento (cuadro 2). Entre 2009 y 2015 pasó del 9,1\% al 10,2\%. Y, de manera simultánea, las transferencias del gobierno nacional han disminuido ligeramente. Las transferencias para inversión se redujeron del 45,2\% de los ingresos totales al 43,4\%. La autonomía fiscal municipal ha mostrado avances, aunque muy tímidos. Las medidas de desempeño fiscal del DNP (2017) muestran que más del 50\% de los municipios están entre las categorías de solventes y sostenibles. Ello significa que pueden realizar todas sus actividades de funcionamiento e inversión sin aprietos financieros.

La estructura de ingresos de los municipios es heterogénea y concentrada en unos pocos. Los de mayor densidad poblacional tienen un nivel de ingresos superior, y una participación más alta de ingresos propios. Muy pocos aprovechan las posibilidades de generar ingresos. La asimetría entre municipios es especialmente notoria cuando se observa el recaudo del impuesto predial. De los $\$ 6,3$ billones que el país recaudó por este concepto en 2015, $\$ 2,4$ billones (el 37,4\%) correspondieron a Bogotá, un monto superior al que recaudaron 1.091 municipios ( $\$ 2,08$ billones). E1 municipio que le sigue es Medellín, con $\$ 0,59$ billones, la cuarta parte del recaudo de Bogotá.

Bogotá ha avanzado de manera significativa en la actualización del catastro, y el avalúo catastral está cerca del $85 \%$ del valor comercial de los inmuebles. Según el Igac, en 2014 el avalúo total del país era de $\$ 1.058$ billones, y el de Bogotá de $\$ 446$ billones (42,2\% del total nacional). El de Medellín era de $\$ 71,8$ billones, el 16,1\% del avalúo de Bogotá.

Para caracterizar los prediales se revisó la tarifa nominal de este impuesto en 973 municipios. En 563 de ellos se compararon las tarifas efectiva y nominal de los predios urbanos. Y en 211 municipios también se calcularon las tarifas nominal y efectiva de los predios rurales. En todos estos el predial es administrado por el Igac. En promedio, la tarifa efectiva es 2,74 inferior a la tarifa nominal.

La gráfica 2 compara las tarifas nominal y efectiva del predial de 21 ciudades. La mayor brecha se presenta en San José de Cúcuta, donde el predial efectivo es del 3\%o y la tarifa nominal de1 9,5\%o. La menor brecha está en Armenia donde la tarifa efectiva es del 6,56\%o y la tarifa nominal del 7\%o. 
En el centro occidente del país el recaudo es mayor. Las capitales del centro occidente recaudaron en un rango intermedio. En municipios de los departamentos de Bolívar, Antioquia, Nariño, Norte de Santander y Chocó predominan tarifas inferiores al 3\%o. Las brechas entre regiones son notables, y como bien señala González (2014), esta falta de convergencia no es nueva, y se observa a todo lo largo del proceso de formación del Estado en Colombia. Hoy, tal como indica la última medición del desempeño integral de los municipios (DNP, 2017), la falta de convergencia es evidente. Hay una relación directa entre el rezago institucional y la baja tarifa efectiva del predial.

\section{Gráfica 2}

Comparación de las tarifas efectiva (\%o) y nominal del impuesto predial 2016 Predios urbanos, 21 ciudades

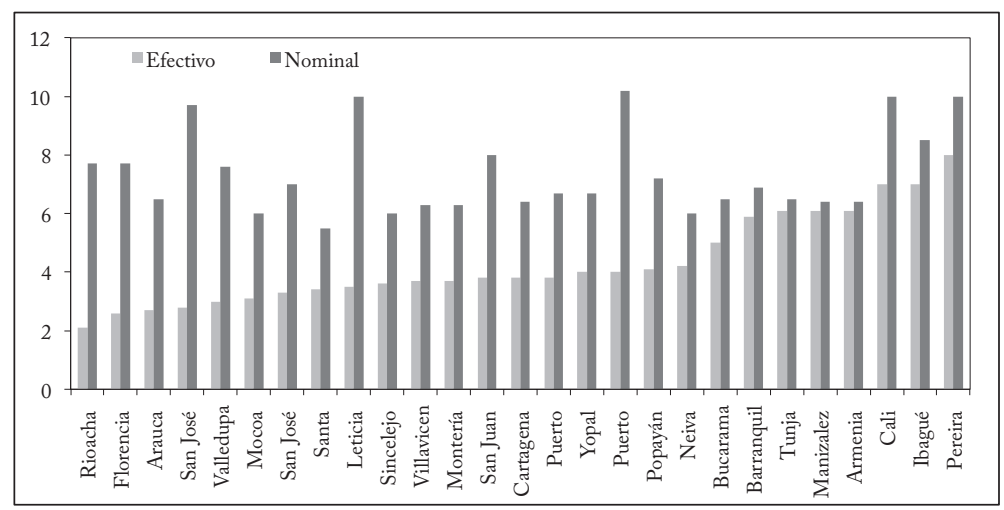

No incluye entidades territoriales con delegación de catastro: Antioquia, Medellín, Bogotá y Cali. Fuente: Igac y estatutos tributarios de cada municipio, cálculos propios.

En los municipios en los que se pudieron comparar las tarifas de predios urbanos y rurales, se encontró que la tarifa efectiva del predial rural es del 2,3\%o, mientras que la de los predios urbanos es del $3,4 \%$. Claramente, la tarifa efectiva de los predios rurales es menor que la tarifa efectiva de los predios urbanos.

No existe una estructura tarifaria homogénea. Cada concejo municipal determina la escala de tarifas sin criterios claros. A veces la tarifa es plana, otras veces trata de responder a un criterio de equidad, pero las categorías que determinan los rangos no se explican ni se justifican bien. Algunos municipios dan énfasis al estrato, otros al avalúo, otros al destino hacendario, otros al tipo de uso y otros al área ${ }^{3}$. En la

${ }^{3}$ La Ley 44 de 1990 ordena a los municipios fijar la tarifa de manera diferenciada y progresiva. Deben tener en cuenta los estratos socioeconómicos, los usos del suelo en el sector urbano, la antigüedad de la formación o 
clasificación, el predio puede ser residencial y no residencial. Y cada categoría tiene tarifas distintas. Si es residencial se puede combinar el estrato con el avalúo. Son seis estratos y en cada estrato puede haber distintas tarifas por rango de avalúo. En la clasificación de predios no residenciales las tarifas se pueden asignar por actividad económica, dotacionales privados o públicos, etc.

Los avalúos y el estrato son aproximaciones imperfectas de la capacidad de pago. En los municipios que lo tienen actualizado, el avalúo es más cercano a la capacidad de pago que el estrato, el cual es la opción menos conveniente. En Colombia, la estratificación comenzó en 1983, con el fin de clasificar las viviendas a partir de sus condiciones físicas aparentes y de su entorno. Una de las razones para recurrir al estrato era la falta de actualización de los avalúos catastrales. Este uso del estrato, que permitió diferenciar y ayudó a ordenar la política de tarifas y subsidios ${ }^{4}$, ya cumplió su tarea y hoy es inadecuado. Ya es hora de replantear este instrumento, y no es conveniente seguir utilizándolo como referente para determinar la progresividad del predial (ONU Habitat, 2016). En Colombia no se ha remplazado el estrato, y no hay un registro adecuado de la capacidad de pago. La forma de hacerlo sería a través de declaración de renta, pero el instrumento hoy es simplificado y tiene poca cobertura. Habría que apuntar a una declaración de renta universal.

Los estratos perdieron su capacidad de clasificación. La mayoría de los hogares están agrupados, en forma incorrecta, en los estratos 2 y 3 . A diferencia de lo que sucede con los estratos, los avalúos sí se han actualizado, sobre todo en Medellín, Bogotá y Cali. Aunque todavía queda un largo camino por recorrer en esta materia, las ciudades principales han hecho avances importantes. El avalúo catastral es un indicador que se aproxima mejor a la capacidad de pago de los hogares que el estrato. Y, además, al avalúo se le pueden fijar tarifas que cumplan los principios de progresividad.

actualización del catastro. A la vivienda popular y a la pequeña propiedad rural se les deben aplicarán las tarifa mínimas. En el caso de los terrenos urbanizables no urbanizados y a los urbanizados no edificados (no se usa el suelo para lo que está dispuesto) el límite superior de la tarifa es el 33\%o. Estas disposiciones son muy generales y no han sido suficientes para ordenar las estructuras tarifarias de los municipios.

${ }^{4}$ En el caso de los servicios públicos se fijó el estrato 4, como el neutro, porque la tarifa paga el costo. Los estratos 5 y 6 contribuyen porque pagan una tarifa superior al costo. $\mathrm{Y}$ los estratos 1,2 y 3 son subsidiados porque pagan una tarifa inferior al costo. 
Es evidente que en cada Concejo el juego político incide en la definición de las tarifas del predial, que en algunos casos son muy bajas. En Bucaramanga, por ejemplo, la tarifa nominal mínima es del 1,5\%o, en Bogotá del 2\%o y en Medellín, Cali y Barranquilla del 4\%o. En municipios de menor categoría hay tarifas mínimas más altas. En San José de Guaviare es del 5\%o, en Leticia del 4,5\% y en Quibdó del 4\%o. No solo hay diversidad en las tarifas, sino también en los criterios para definir las escalas (Bonilla, 2014). También se observa heterogeneidad en las tarifas máximas. La única ciudad que utiliza el límite superior es Cúcuta, con el 16\%o. Le siguen Medellín y Chinchiná, con el 15,5\% y el 15\%o respectivamente. En Bogotá la tarifa es del $11 \%$.

Entre las razones que explican la baja tarifa efectiva se pueden mencionar las siguientes: a) cobro ineficiente por razones políticas o gerenciales; b) incapacidad administrativa del municipio que no tiene información del valor de los avalúos ni del área de la propiedad. Además, el municipio no tiene mapas de los predios, ni actualiza su base de datos a medida que se construyen o modifican los inmuebles. Por falta de información, el municipio hace una propuesta de auto avalúo inadecuada; c) incapacidad para sancionar a los morosos, y d) interpretación laxa de la ley a favor del propietario.

La Comisión de Expertos para la Equidad y la Competitividad Tributaria (2015) incluye otros factores que pueden incidir en el bajo recaudo: 1) no existe una regla clara para expedir la liquidación del impuesto. En Bogotá, por ejemplo, los contribuyentes pueden hacerlo mediante auto avalúo. Presentan una declaración en la que se autoliquida el impuesto, pero el Distrito les remite la liquidación sugerida. El procedimiento varía en los demás municipios. Algunos facturan, otros emiten recibos y otros le cobran a quien se acerca a pagar. Esta falta de precisión en la información es una de las causas de la baja tributación efectiva; 2) mala gestión, que se refleja en elevadas carteras por cobrar; 3) el procedimiento para liquidar y notificar el monto de la obligación tributaria no está suficientemente reglamentado, y 4) no se conoce el término de prescripción de la obligación y, por tanto, existe una cartera incierta.

Si se compara el recaudo de Colombia como proporción del PIB con el promedio de los países de la Ocde (2017), se observa una gran brecha. En 2014, el recaudo por impuesto a la propiedad en Colombia fue del 0,75\% del PIB, mientras que los países miembros de la Ocde recaudaron, en promedio, el 1,86\% de su PIB. E1 mayor recaudo se observa en Francia, con el 3,88\%. Le siguen Canadá y Bélgica, con 
recaudos del 3,67\% y del 3,56\%, respectivamente. Aunque en los países de la Ocde los impuestos a la propiedad son más altos que en Colombia, la Ocde insiste en la necesidad de aumentarlos, rescatando propuestas similares de autores como Bird y Slack (2002). La cifra de Colombia solo es comparable con las de México y Chile, que recaudaron el 0,83\% y el 0,32\%, como porcentaje del PIB.

\section{MODERNIZACIÓN DEL CATASTRO, RELACIÓN INVERSA Y PRODUCTIVIDAD}

Para mejorar la dinámica agropecuaria, la eficiencia se debe combinar, un buen uso de la tierra -acorde con su vocación-con un apoyo estatal a la pequeña propiedad. Para lograr ambos objetivos se requiere una adecuada información catastral, así un diseño tributario adecuado que castigue las tierras ociosas y estimule la productividad.

\section{LA CONJUNCIÓN DE LA VOCACIÓN Y EL USO DEL SUELO}

La adecuada tributación de la tierra depende de que la información esté disponible en forma oportuna. Es decir, está ligada al buen funcionamiento del catastro. Existe una relación directa entre la actualización catastral y un mercado de tierras eficiente. El catastro es más que un instrumento fiscal, "su propósito es amplio, ya que se trata de un sistema de información de tierras indispensable para el desarrollo social y para la consolidación del mercado del suelo"(Bonilla y González, 2016, p. 50).

E1 Estado colombiano tiene muy poca información sobre el uso del suelo y de los precios. En las condiciones actuales no logra hacer un ordenamiento territorial adecuado. Si el Estado tuviese certeza de la estructura de la propiedad podría ejercer control y hacer uso apropiado de herramientas como el impuesto predial.

Uno de los grandes problemas es la desactualización catastral. Según el documento Conpes 3859 (DNP, 2016), el 32,3\% de los predios del país estaba sin actualización catastral en 2016. En la zona urbana, la desactualización era del $23 \%$ y en la zona rural del 59\%. Están actualizados los catastros descentralizados de Bogotá, Medellín y Cali. El catastro administrado por el Igac está desactualizado en un 43,6\%, y el de Antioquia en un 42\%. Los gobiernos no le han dado al Igac la importancia estratégica que merece, y no se le asigna un presupuesto acorde con la relevancia de sus funciones. En los acuerdos de La Habana el gobierno se comprometió a adoptar e implementar el catastro multipropósito rural-urbano. E1 
documento Conpes 3859, que especifica el compromiso, hace un autodiagnóstico del catastro y reconoce que la capacidad institucional es muy limitada.

Los avalúos catastrales no reflejan las características físicas y económicas de los inmuebles. Al contrastarlos con el valor comercial se observan diferencias del 66\% en las zonas urbanas y hasta del 99\% en las rurales. El catastro descentralizado de Bogotá, Medellín y Cali está muy actualizado, y los avalúos se acercan al precio comercial de los predios. El catastro centralizado, administrado por el Igac, está rezagado. Es conveniente, entonces, delegar el catastro y que el Igac lo vaya entregando a los municipios con mayor capacidad institucional para administrarlo.

No hay una representación precisa de los predios. La cartografía básica del país no cumple los requerimientos de escala. Tampoco hay estándares bien definidos para el levantamiento catastral, lo que genera inconsistencias en la ubicación de los predios. A esto se suma la insuficiencia de profesionales calificados para realizar levantamientos catastrales. Hay profesiones y especializaciones que se ajustan al perfil, pero no hay manera de certificar que los profesionales tienen las competencias y conocimientos necesarios.

No hay buena interrelación entre las instancias que producen y administran la información de tierras. En particular, no hay coordinación entre las autoridades catastrales y la Superintendencia de Notariado y Registro. E1 resultado directo de este cuello de botella es que la información catastral no coincide con la del registro público de propiedad. A marzo de 2015, de 32.932.119 de predios rurales registrados en el catastro del Igac, solo el $46 \%$ pudo asociarse a su folio de matrícula inmobiliaria (DNP, 2016). La información de catastro $\mathrm{y}$ registro de un mismo inmueble difiere porque los procedimientos jurídicos de cada instancia no tienen relación entre sí y no se validan mutuamente ${ }^{5}$.

El catastro es un instrumento adecuado para dirimir conflictos de uso del suelo. En las diferentes regiones del país hay actores que tienen intereses muy diferentes sobre un mismo territorio. Bonilla y González (2016) observan que el Estado no tiene claridad sobre la manera de clasificar el suelo del país. Analizan las categorías que utilizan las entidades y la información que existe en el territorio, y muestran que los criterios para clasificar los suelos son heterogéneos,

${ }^{5}$ La información de registro proviene de escrituras públicas, actos administrativos o sentencias, mientras que la información de catastro es producto de visitas y observación (ibíd.).

Revista de Economía Institucional, vol. 20, N. ${ }^{\circ}$ 39, Segundo Semestre/2oi 8, pp. 2 it-229 
y ello lleva a traslapes que complican el estudio del conflicto de usos. Por ejemplo, mientras que una entidad dice que cierta área tiene vocación agrícola, otra afirma que la vocación es forestal.

Colombia tiene 114 millones de ha y, según información de la Upra (2014), unos 32 millones de ha no tienen un uso adecuado (el $30 \%$ del territorio nacional). Se sobre utilizan 18,3 millones de ha (el 17\%), mientras que se subutilizan 14,9 millones (el 13\%). Existen 15 millones de ha con vocación agrícola, pero solo se usan 6 millones para ese propósito. Además, solo 8 millones de ha tienen vocación ganadera, pero se utilizan 38 millones en pastoreo.

Según el Igac (2012), el 13\% de los suelos con vocación agrícola se destina a otros usos, lo que genera ineficiencias productivas, daños ambientales y obliga a los pequeños campesinos a expandir la frontera agrícola en laderas y bosques. La Misión para la Transformación del Campo (Ocampo, 2015) observa que entre 2000 y 2009 el área de producción agrícola aumentó en un 9,3\%. Sin embargo, este incremento ocurrió en municipios con escasa densidad rural, baja competitividad y alta violencia. También aumentó en áreas de bosque, donde hay dificultades para la provisión de bienes públicos y no existen las mejores condiciones de competitividad. La Misión también observó que algunas zonas urbanas crecen en forma dispersa o con baja densidad. La ampliación de las aglomeraciones eleva el precio del suelo rural y encarece la producción de alimentos cerca de las ciudades. Un buen ordenamiento territorial debería crear corredores agrícolas alrededor de las ciudades. En estas áreas el precio del suelo no sube al mismo ritmo que en las zonas urbanas, y ello permite que la agricultura sea rentable.

\section{LA RELACIÓN INVERSA}

El ordenamiento del territorio, junto con un sistema tributario que castigue la ineficiencia y mejore la equidad, ayudaría a modernizar el agro. La predominancia de la pequeña producción abre el espacio para reflexionar sobre la relación inversa entre productividad media por hectárea y tamaño de la explotación. En palabras de Berry:

Dos características generales (casi universales) son "la relación inversa" entre el tamaño de la explotación y la productividad de la tierra, y la relación positiva entre el tamaño de esta explotación y la productividad de la mano de obra. Estas dos relaciones señalan una tercera correspondencia de carácter universal: la razón "mano de obra/tierra" disminuye con el tamaño de la explotación, típicamente en forma dramática. (Berry, 2017, p. 2) 
Esta hipótesis de Albert Berry se cumple perfectamente en el caso colombiano (cuadro 3). Las UPA agrícolas de menos de 1 ha tienen una productividad de 8 toneladas por ha. En las UPA de más de 10.000 ha, es de 3,9. Ello significa que en la pequeña unidad la productividad es casi el doble que en las UPA de mayor tamaño. En las de 20 a 500 ha la productividad es relativamente buena $(6,1$ ton/ha) aunque menor que en las unidades pequeñas. La diferencia es más significativa en el sector pecuario. Mientras que en las fincas pequeñas la productividad media es de 8,8 ton/ha, en las UPA grandes apenas llega a 0,2 ton/ha. Esta diferencia tiene que ver con el crecimiento de la ganadería extensiva. En las UPA forestales la productividad no parece estar relacionada con el tamaño de la unidad productiva.

Cuadro 3

Productividad media y tamaño de la explotación (Hectáreas y toneladas por hectárea)

\begin{tabular}{lccc}
\hline Tamaño & Agrí́cola & Forestal & Pecuario \\
\hline Menos 1 & 8,0 & 2,0 & 8,8 \\
$1-3$ & 5,1 & 1,9 & 2,8 \\
$3-20$ & 5,8 & 1,6 & 1,3 \\
$20-500$ & 6,1 & 1,8 & 0,5 \\
$500-10.000$ & 4,2 & 2,5 & 0,2 \\
Más 10.000 & 3,9 & 0,8 & 0,0 \\
\hline
\end{tabular}

Fuente: CNA 2014, cálculos de la Upra.

Durante mucho tiempo la intuición sobre la relación inversa entre tamaño del predio y productividad estuvo presente en la discusión pública. El trabajo de Sen (1962) sobre la estructura agrícola en India hizo tres grandes observaciones basadas en información empírica. Primera, el trabajo familiar es parte de la actividad doméstica y, entonces, para conocer la estructura de costos hace falta información sobre los salarios. Segunda, la rentabilidad de la agricultura aumenta con el monto de la inversión. Tercera, la productividad por unidad de superficie disminuye con el tamaño de la explotación.

Berry y Carter han mostrado la relación inversa entre el tamaño de los predios y su nivel de productividad en otros países en desarrollo. Berry (1983) usó información secundaria (de encuestas y censal) para analizar los rendimientos de escala de la tierra en Brasil, Colombia, Filipinas y Malasia. En Colombia, Berry usó información del censo de 1960. Sus análisis mostraron que los predios de 3 a 5 ha producían un $15 \%$ más de valor agregado por hectárea que los de más de 500 ha. Según Berry, el único factor que explicó estas diferencias fue la 
rápida caída de la proporción del área cultivada en el área total del predio, a medida que el tamaño del predio aumentaba.

Por su parte, Carter (1984) hizo un análisis de la producción agrícola campesina en el estado de Haryana, en India, con información de encuestas realizadas entre 1969 y 1972. Después de controlar los datos por problemas de recolección de información y sesgo de selección, encontró una clara relación inversa entre el tamaño de las granjas y su nivel de productividad. No obstante, advirtió que las granjas pequeñas subutilizan sus factores de producción y, en términos generales, que no se maximiza la utilidad de los campesinos.

Trabajos recientes encuentran tendencias similares en el caso colombiano. El informe de desarrollo humano de las Naciones Unidas (PNUD, 2011) señala que una de las razones por las que el modelo que se aplica al sector rural ha fracasado es la insistencia en favorecer la producción extensiva. En general, no tiene en cuenta las ventajas de productividad que tienen los predios pequeños.

La Misión para la Transformación del Campo también hizo algunas consideraciones específicas sobre las pequeñas propiedades: "la pequeña producción no tiene solamente beneficios sociales, sino que suele ser más productiva y, por lo tanto, puede contribuir a la competitividad del país" (Ocampo, 2015, p. 146).

Berry (2010) muestra que cuando una sociedad busca aumentar el nivel y la distribución del ingreso, la pequeña propiedad of rece ventajas por la relación inversa entre tamaño y productividad. En las zonas rurales hay trabajo abundante, lo que significa que las fincas lo usan en forma intensiva. Por ejemplo, si hay suma abundancia de trabajo, los habitantes no pueden conseguir empleos bien remunerados en otras actividades; así, el costo de oportunidad es cero. Otra ventaja es que quienes laboran en pequeñas propiedades pueden tener más incentivos para trabajar con mayor intensidad. Además, si son propietarios, se esfuerzan más en el proceso de transformación. Y su cercanía al campo les proporciona un conocimiento privilegiado que se puede reflejar en mayor productividad. Por otro lado, los predios pequeños se especializan en alimentos básicos, y ello favorece la seguridad alimentaria y la provisión de servicios ambientales.

Para que la pequeña propiedad logre aprovechar sus ventajas es necesario el apoyo del Estado. Uno de los instrumentos que puede emplear para darle apoyo es un sistema tributario diseñado que favorezca la equidad y la eficiencia. Esta medida fiscal deben ir acompañadas de la oferta adecuada de bienes y servicios, como proponen las Naciones Unidas (PNUD, 2011) y La Misión para la Transformación 
del Campo (Ocampo, 2105): asistencia técnica, educación y salud, crédito blando, vías de comunicación y un largo etcétera.

\section{REFERENCIAS BIBLIOGRÁFICAS}

Arteaga, J., Castro, C. et al. (2017). Fondo de tierras del acuerdo agrario de La Habana: estimación y propuestas alternativas. Documento Cede 41, 1-106.

Banco Mundial. (2004). Colombia: una política de tierras en transición. Documento Cede 2004-29, 1-96.

Berry, A. (1983). Agrarian structure, rural labour markets, and trends in rural incomes in Latin America. En V. L. Urquidi y S. Trejo (eds.), Human resources, employment and development, vol. 4: Latin America (pp.174-194). Londres: MacMillan.

Berry, A. (2010). The economics of land reform and of small farms in developing countries: Implications for post-conflict situations. En M. Bergsmon, C. Rodríguez et al. (eds.). Distributive justice in transitions (pp. 25-70). Oslo: Torkel Opsahl Academic EPublisher.

Berry, A. (2017). Avance y fracaso en el agro colombiano, siglos XX y XXI. Bogotá: Universidad del Rosario.

Bird, R. y Slack, E. (2002). Land and property taxation: A review. Cambridge: Edward Elgar Publishing Limited.

Bonilla, E. (2014). La cultura tributaria como herramienta de política fiscal: la experiencia de Bogotá. Revista Ciudades, Estados y Politica, 1(1), 21-35.

Bonilla, E. C. y González, J. I. (coord.). (2016). Aproximación al mercado de tierras en Colombia. Bogotá: Universidad Nacional, Planeta.

Carter, M. (1984). Identification of the inverse relationship between farm size and productivity: An empirical analysis of peasant agricultural production. Oxford Economic Papers, 36(1), 131-145.

Comisión de expertos para la equidad y la competitividad tributaria. (2015). Informe final presentado al Ministro de Hacienda y Crédito Público. Bogotá: Ministerio de Hacienda, Fedesarrollo.

Corbacho, A., Fretes, V. et al. (2013). Recaudar no basta. Los impuestos como instrumento de desarrollo. Washington D.C: BID.

DNP. (2016). Política para la adopción e implementación de un catastro multipropósito rural-urbano. Documento Conpes 3859.

DNP. (2017). Guía orientaciones para realizar la medición del desempeño integral municipal. Guía para oficinas departamentales de planeación, Bogotá: DNP.

George, H. (1881). The land question and related writings. Viewpoint and counterviewpoint on the need for land reform. Nueva York: Robert Schalkenbach Foundation.

González, F. (2014). Poder y violencia en Colombia. Bogotá: Odecofi, Cinep.

Hotelling, H. (1931). The economics of exhaustible resources. Journal of Political Economy, 39(2), 137-175.

Igac. (2012). Sistema de información geográfica de Colombia. Bogotá: Sigot, Igac. 
Machado, A. (2009). La reforma rural. Una deuda social y politica. Bogotá: CID, Universidad Nacional de Colombia.

Marx, K. (1968). El Capital [1867], 3 vol. México DF: Fondo de Cultura Económica.

Mill, J. S. (1978). Principios de economía politica [1885]. México DF: Fondo de Cultura Económica.

Ocampo, J. (dir). (2015). El campo colombiano: un camino hacia el bienestar y la paz. Informe detallado de la misión para la transformación del campo, 3 tomos. Bogotá: DNP.

ONU habitat. (2016). Propuesta de implementación de un nuevo sistema de asignación de subsidios y contribuciones en servicios públicos domiciliarios. Informe final. Bogotá: ONU Habitat.

Ocde. (2017). Estudios económicos de la Ocde. Colombia. París: Ocde.

PNUD. (2011). Colombia rural. Razones para la esperanza. Informe nacional de desarrollo bumano 2011. Bogotá: PNUD.

Ricardo, D. (2001). On the principles of political economy and taxation [1817]. Kitchener. Ont.: Batoche Books.

Sen, A. (1962). An aspect of Indian agriculture. Economic Weekly, febrero, 243-246.

Smith, A. (1994). Investigación sobre la naturaleza y causas de la riqueza de las naciones [1776]. Madrid: Alianza.

Stiglitz, J. (2015). New theoretical perspectives on the distribution of income and wealth among individuals: Part IV: Land and credit. NBER working paper 21192, Cambridge. Mass.: NBER.

Upra. (2014). Bases conceptuales procesos de regularización de la propiedad rural y acceso a tierras. Bogotá: Upra. 